JOURNAL OF THE

AMERICAN MATHEMATICAL SOCIETY

Volume 20, Number 3, July 2007, Pages 603-628

S 0894-0347(07)00555-3

Article electronically published on February 6, 2007

\title{
ON THE SINGULARITY PROBABILITY OF RANDOM BERNOULLI MATRICES
}

\author{
TERENCE TAO AND VAN VU
}

\section{INTRODUCTION}

Let $n$ be a large integer, and let $M_{n}$ be a random $n \times n$ matrix whose entries are i.i.d. Bernoulli random variables (each entry is \pm 1 with probability $1 / 2$ ). In this paper we consider the well-known question of estimating $P_{n}:=\mathbf{P}\left(\operatorname{det}\left(M_{n}\right)=0\right)$, the probability that $M_{n}$ is singular.

By considering the event that two columns or two rows of $M_{n}$ are equal (up to sign), it is clear that

$$
P_{n} \geq(1+o(1)) n^{2} 2^{1-n} .
$$

Here and in the sequel we use the asymptotic notation under the assumption that $n$ tends to infinity. It has been conjectured by many researchers that in fact

\section{Conjecture 1.1.}

$$
P_{n}=(1+o(1)) n^{2} 2^{1-n}=\left(\frac{1}{2}+o(1)\right)^{n} .
$$

In a breakthrough paper [11, Kahn, Komlós and Szemerédi proved that

$$
P_{n}=O\left(.999^{n}\right) .
$$

In a recent paper [17 we improved this bound slightly to $O\left(.958^{n}\right)$. The main result of this paper is the following more noticeable improvement.

\section{Theorem 1.2.}

$$
P_{n} \leq\left(\frac{3}{4}+o(1)\right)^{n} .
$$

The proof of this theorem uses several ideas from [11, 17]. The key new ingredient is the applications of Freiman-type inverse theorems and other tools from additive combinatorics.

In the next section, we present the initial steps of the proof of Theorem 1.2 , These steps will reveal the origin of the bound $\left(\frac{3}{4}+o(1)\right)^{n}$ and also provide an approach to the conjectured bound $(1+o(1)) n^{2} 2^{1-n}$.

Received by the editors November 5, 2004.

2000 Mathematics Subject Classification. Primary 15A52.

The first author is a Clay Prize Fellow and is supported by a grant from the Packard Foundation.

The second author is an A. Sloan Fellow and is supported by an NSF Career Grant. 


\section{Reduction to MEdium COMBinatorial Dimension}

We begin the proof of Theorem 1.2. We first make a convenient (though somewhat artificial) finite field reduction. Observe that any $n \times n$ matrix with entries \pm 1 can have determinant at most $n^{n / 2}$ (since the magnitude of the determinant equals the volume of a $n$-dimensional parallelopiped, all of whose edges have length $\sqrt{n})$; this is also known as Hadamard's inequality. Thus if we introduce a finite field $F:=\mathbf{Z} / p \mathbf{Z}$ of prime order

$$
|F|=p>n^{n / 2}
$$

then $P_{n}$ is also the probability that a random $n \times n$ matrix with entries \pm 1 (now thought of as elements of $F$ ) is singular in the finite field $F$. Henceforth we fix the finite field $F$ and shall work over the finite field $F$ instead of over R. In particular, linear algebra terminology such as "dimension", "rank", "linearly independent", "subspace", "span", etc., will now be with respect to the field F. One advantage of this discretized setting is that all collections of objects under consideration will now automatically be finite, and one can perform linear change of variables over $F$ without having to worry about Jacobian factors. Note that while one could use Bertrand's postulate to fix $|F|$ to be comparable to $n^{n / 2}$, we will not need to do so in this paper. Indeed it will be more convenient to take $|F|$ to be extremely large (e.g. larger than $\exp (\exp (C n)))$ to avoid any "torsion" issues.

Now let $\{-1,1\}^{n} \subset F^{n}$ be the discrete unit cube in $F^{n}$. We let $X$ be the random variable taking values in $\{-1,1\}^{n}$ which is distributed uniformly on this cube (thus each element of $\{-1,1\}^{n}$ is attained with probability $2^{-n}$ ). Let $X_{1}, \ldots, X_{n} \in$ $\{-1,1\}$ be $n$ independent samples of $X$. Then

$$
P_{n}:=\mathbf{P}\left(X_{1}, \ldots, X_{n} \text { linearly dependent }\right) .
$$

For each linear subspace $V$ of $F^{n}$, let $A_{V}$ denote the event that $X_{1}, \ldots, X_{n}$ span $V$. Let us call a space $V$ non-trivial if it is spanned by the set $V \cap\{-1,1\}^{n}$. Note that $\mathbf{P}\left(A_{V}\right) \neq 0$ if and only if $V$ is non-trivial. Since every collection of $n$ linearly dependent vectors in $F^{n}$ will span exactly one proper subspace $V$ of $F^{n}$, we have

$$
P_{n}=\sum_{V \text { a proper non-trivial subspace of } F^{n}} \mathbf{P}\left(A_{V}\right) .
$$

In [17. Lemma 5.1] (see also [1] ), we showed that the dominant contribution to this sum came from the hyperplanes:

$$
P_{n}=2^{o(n)} \sum_{V \text { a non-trivial hyperplane in } F^{n}} \mathbf{P}\left(A_{V}\right) .
$$

Thus it will suffice to show that

$$
\sum_{V \text { a non-trivial hyperplane in } F^{n}} \mathbf{P}\left(A_{V}\right) \leq(3 / 4+o(1))^{n} .
$$

We remark that the arguments below also extend to control the lower-dimensional spaces directly, but it will be somewhat simpler technically to work just with hyperplanes.

As in [11, the next step is to partition the non-trivial hyperplanes $V$ into a number of classes, depending on a quantity which we shall call the combinatorial dimension. 
Definition 2.1 (Combinatorial dimension). Let $D:=\left\{d_{ \pm} \in \mathbf{Z} / n: 1 \leq d_{ \pm} \leq n\right\}$. For any $d_{ \pm} \in D$, we define the combinatorial Grassmannian $\operatorname{Gr}\left(d_{ \pm}\right)$to be the set of all non-trivial hyperplanes $V$ in $F^{n}$ with

$$
2^{d_{ \pm}-1 / n}<\left|V \cap\{-1,1\}^{n}\right| \leq 2^{d_{ \pm}} .
$$

We will refer to $d_{ \pm}$as the combinatorial dimension of $V$.

Remark 2.2. Following [11, we have gradated the combinatorial dimension in steps of $1 / n$ rather than steps of 1 . This is because we will need to raise probabilities such as $\mathbf{P}(X \in V)$ to the power $n$, and thus errors of $2^{O(1 / n)}$ will be acceptable while errors of $2^{O(1)}$ can be more problematic. This of course increases the number of possible combinatorial dimensions from $O(n)$ to $O\left(n^{2}\right)$, but this will have a negligible impact on our analysis.

It thus suffices to show that

$$
\sum_{d_{ \pm} \in D} \sum_{V \in \operatorname{Gr}\left(d_{ \pm}\right)} \mathbf{P}\left(A_{V}\right) \leq\left(\frac{3}{4}+o(1)\right)^{n} .
$$

It is therefore of interest to understand the size of the combinatorial Grassmannians $\operatorname{Gr}\left(d_{ \pm}\right)$and of the probability of the events $A_{V}$ for hyperplanes $V$ in those Grassmannians.

There are two easy cases, one when $d_{ \pm}$is fairly small and one where $d_{ \pm}$is fairly large, both of which were treated in [11, and which we present below.

Lemma 2.3 (Small combinatorial dimension estimate [11). Let $0<\alpha<1$ be arbitrary. Then

$$
\sum_{d_{ \pm} \in D: 2^{d^{ \pm}-n} \leq \alpha^{n}} \sum_{V \in \operatorname{Gr}\left(d_{ \pm}\right)} \mathbf{P}\left(A_{V}\right) \leq n \alpha^{n} .
$$

Proof. Observe that if $X_{1}, \ldots, X_{n}$ span $V$, then there are $n-1$ vectors among the $X_{i}$ which already span $V$. By symmetry, we thus have

$$
\mathbf{P}\left(A_{V}\right)=\mathbf{P}\left(X_{1}, \ldots, X_{n} \text { span } V\right) \leq n \mathbf{P}\left(X_{1}, \ldots, X_{n-1} \text { span } V\right) \mathbf{P}(X \in V) .
$$

On the other hand, if $V \in \operatorname{Gr}\left(d_{ \pm}\right)$and $2^{d_{ \pm}-n} \leq \alpha^{n}$, then $\mathbf{P}(X \in V) \leq \alpha^{n}$ thanks to (4). Thus we have

$$
\mathbf{P}\left(A_{V}\right) \leq n \alpha^{n} \mathbf{P}\left(X_{1}, \ldots, X_{n-1} \text { span } V\right) .
$$

Since $X_{1}, \ldots, X_{n-1}$ can span at most one space $V$, the claim follows.

Lemma 2.4 (Large combinatorial dimension estimate [11]). We have

$$
\sum_{d_{ \pm} \in D: 2^{d_{ \pm}-n} \geq 100 / \sqrt{n}} \sum_{V \in \operatorname{Gr}\left(d_{ \pm}\right)} \mathbf{P}\left(A_{V}\right) \leq(1+o(1)) n^{2} 2^{-n} .
$$

Proof. (Sketch) For this proof it is convenient to work back in Euclidean space $\mathbf{R}^{n}$ instead of in the finite field model $F^{n}$; observe that one can identify nontrivial hyperplanes in $F^{n}$ with non-trivial hyperplanes in $\mathbf{R}^{n}$ without affecting the combinatorial dimension. Let $V \subset \mathbf{R}^{n}$ be a hyperplane in $\operatorname{Gr}\left(d_{ \pm}\right)$with $2^{d_{ \pm}-n} \geq$ $\frac{100}{\sqrt{n}}$. Then by (4) we have

$$
\mathbf{P}(X \in V) \geq 50 / \sqrt{n}
$$


(say). Since $V$ is spanned by elements $V \cap\{-1,1\}^{n}$ which lie in the integer lattice $\mathbf{Z}^{n}$, the orthogonal complement $V^{\perp} \subset \mathbf{R}^{n}$ contains at least one integer vector $a=\left(a_{1}, \ldots, a_{n}\right) \in \mathbf{Z}^{n} \backslash\{0\}$ which is not identically zero. In particular we see that

$$
\mathbf{P}\left(a_{1} X_{1}+\ldots+a_{n} X_{n}\right) \geq 50 / \sqrt{n} .
$$

But by Erdös's Littlewood-Offord inequality (see [5]) this forces at most $n / 2$ (say) of the coefficients $a_{1}, \ldots, a_{n}$ to be non-zero, if $n$ is sufficiently large. Thus

$$
\begin{aligned}
& \sum_{d_{ \pm} \in D: 2^{d} \pm^{-n} \geq \frac{100}{\sqrt{n}}} \sum_{V \in \operatorname{Gr}\left(d_{ \pm}\right)} \mathbf{P}\left(A_{V}\right) \\
& \leq \mathbf{P}\left(a \cdot X_{1}=\ldots=a \cdot X_{n}=0 \text { for some } a \in \Omega\right)
\end{aligned}
$$

where $\Omega$ is the set of those vectors $a=\left(a_{1}, \ldots, a_{n}\right) \in \mathbf{Z}^{n} \backslash\{0\}$ which have at most $n / 2$ non-zero entries. But in [11, Section 3.1] it is shown that

$$
\mathbf{P}\left(a \cdot X_{1}=\ldots=a \cdot X_{n}=0 \text { for some } a \in \Omega\right) \leq(1+o(1)) n^{2} 2^{-n}
$$

and the claim follows. (In fact, in the estimate in [11] one can permit as many as $n-3 \log _{2} n$ of the coefficients $a_{1}, \ldots, a_{n}$ to be non-zero).

We will supplement these two easy lemmas with the following, somewhat more difficult, result.

Proposition 2.5 (Medium combinatorial dimension estimate). Let $0<\epsilon_{0} \ll 1$, and let $d_{ \pm} \in D$ be such that $\left(\frac{3}{4}+2 \epsilon_{0}\right)^{n}<2^{d_{ \pm}-n}<\frac{100}{\sqrt{n}}$. Then we have

$$
\sum_{V \in \operatorname{Gr}\left(d_{ \pm}\right)} \mathbf{P}\left(A_{V}\right) \leq o(1)^{n}
$$

where the rate of decay in the o(1) quantity depends on $\epsilon_{0}$ (but not on $d_{ \pm}$).

Note that $D$ has cardinality $|D|=O\left(n^{2}\right)$. Thus if we combine this proposition with Lemma 2.3 (with $\alpha:=\frac{3}{4}+2 \epsilon_{0}$ ) and Lemma 2.4, we see that we can bound the left-hand side of (5) by

$$
n\left(\frac{3}{4}+2 \epsilon_{0}\right)^{n}+n^{2} o(1)^{n}+(1+o(1)) n^{2} 2^{-n}=\left(\frac{3}{4}+2 \epsilon_{0}+o(1)\right)^{n} .
$$

Since $\epsilon_{0}$ is arbitrary, Theorem 1.2 follows.

It thus suffices to prove Proposition 2.5. This we shall do in later sections, but for now let us just remark that this argument suggests a way to obtain Conjecture 1.1 in full. A comparison of the three bounds in Lemma 2.3, Lemma 2.4, and Proposition 2.5 reveals that it is the estimate in Lemma 2.3 which is by far the most inefficient. Indeed, from the other two lemmas we now see that the conjecture $P_{n}=\left(\frac{1}{2}+o(1)\right)^{n}$ is equivalent to proving that

$$
\sum_{d_{ \pm} \in D: 2^{d_{ \pm}-n} \leq\left(\frac{3}{4}+2 \epsilon_{0}\right)^{n}} \sum_{V \in \operatorname{Gr}\left(d_{ \pm}\right)} \mathbf{P}\left(A_{V}\right) \leq\left(\frac{1}{2}+o(1)\right)^{n}
$$

for at least one value of $\epsilon_{0}>0$. This bound is sharp (except for the $o(1)$ factor) as can be seen by considering the event that two of the $X_{i}$ are equal up to sign. On the other hand, if we let $B$ denote the event that $a_{1} X_{1}+\ldots+a_{n} X_{n}=0$ for some $a_{1}, \ldots, a_{n} \in \mathbf{Z}^{n} \backslash\{0\}$ with at most $n-3 \log _{2} n$ non-zero entries, it is shown in [11, 
Section 3.1] that $\mathbf{P}(B)=(1+o(1)) n^{2} 2^{-n}$ (this is basically the transpose of Lemma 2.4). Thus it would suffice to show

$$
\sum_{d_{ \pm} \in D: 2^{d_{ \pm}-n} \leq\left(\frac{3}{4}+2 \epsilon_{0}\right)^{n}} \sum_{V \in \operatorname{Gr}\left(d_{ \pm}\right)} \mathbf{P}\left(A_{V} \backslash B\right)=\left(\frac{1}{2}+o(1)\right)^{n} .
$$

Based on Proposition 2.5. we may tentatively conjecture that in fact we have the stronger statement

$$
\sum_{d_{ \pm} \in D: 2^{d_{ \pm}}-n} \sum_{V \in\left(\frac{3}{4}+2 \epsilon_{0}\right)^{n}} \mathbf{P}\left(A_{V} \backslash B\right)=o(1)^{n}
$$

this for instance would even imply the stronger conjecture in Conjecture 1.1. But our Fourier-based methods seem to hit a natural limit at $2^{d_{ \pm}-n} \sim(3 / 4)^{n}$ and so we are unable to obtain any further progress towards (7).

\section{The general APPROACH}

We now informally discuss the proof of Proposition 2.5, the rigorous proof will begin in Section 4. We start with the trivial bound

$$
\sum_{V \in \operatorname{Gr}\left(d_{ \pm}\right)} \mathbf{P}\left(A_{V}\right) \leq 1
$$

that arises simply because any vectors $X_{1}, \ldots, X_{n}$ can span at most one space $V$. To improve upon this trivial bound, the key innovation in [11] is to replace $X$ by another random variable $Y$ which tends to be more concentrated on subspaces $V$ than $X$ is. Roughly speaking, one seeks the property

$$
\mathbf{P}(X \in V) \leq c \mathbf{P}(Y \in V)
$$

for some absolute constant $0<c<1$ and for all (or almost all) subspaces $V \in$ $\operatorname{Gr}\left(d_{ \pm}\right)$. From this property, one expects (heuristically, at least) that

$$
\mathbf{P}\left(A_{V}\right)=\mathbf{P}\left(X_{1}, \ldots, X_{n} \text { span } V\right) \leq c^{n} \mathbf{P}\left(Y_{1}, \ldots, Y_{n} \text { span } V\right),
$$

where $Y_{1}, \ldots, Y_{n}$ are i.i.d. samples of $Y$, and then by applying the trivial bound (8) with $Y$ instead of $X$, we would then obtain a bound of the form $\sum_{V \in \operatorname{Gr}\left(d_{ \pm}\right)} \mathbf{P}\left(A_{V}\right) \leq$ $c^{n}$, at least in principle. Clearly, it will be desirable to make $c$ as small as possible; if we can make $c$ arbitrarily small, we will have established Proposition 2.5.

The random variable $Y$ can be described as follows. Let $0 \leq \mu \leq 1$ be a small absolute constant (in [1] the value $\mu=\frac{1}{108} e^{-1 / 108}$ was chosen), and let $\eta^{(\mu)}$ be a random variable taking values in $\{-1,0,1\} \subset F$ which equals 0 with probability $1-\mu$ and equals +1 or -1 with probability $\mu / 2$ each. Then let $Y:=$ $\left(\eta_{1}^{(\mu)}, \ldots, \eta_{n}^{(\mu)}\right) \in F^{n}$, where $\eta_{1}^{(\mu)}, \ldots, \eta_{n}^{(\mu)}$ are i.i.d. samples of $\eta^{(\mu)}$. By using some Fourier-analytic arguments of Halász, a bound of the form

$$
\mathbf{P}(X \in V) \leq C \sqrt{\mu} \mathbf{P}(Y \in V)
$$

was shown in [11, where $C$ was an absolute constant (independent of $\mu$ ) and $V$ was a hyperplane which was non-degenerate in the sense that its combinatorial dimension was not too close to $n$. For $\mu$ sufficiently small, one then obtains (9) for some $0<c<1$, although one cannot make $c$ arbitrarily small without shrinking $\mu$ also.

There are however some technical difficulties with this approach, arising when one tries to pass from (9) to (10). The first problem is that the random variable 
$Y$, when conditioned on the event $Y \in V$, may concentrate on a lower-dimensional subspace on $V$, making it unlikely that $Y_{1}, \ldots, Y_{n}$ will span $V$. In particular, $Y$ has a probability of $(1-\mu)^{n}$ of being the zero vector, which basically means that one cannot hope to exploit (9) in any non-trivial way once $\mathbf{P}(X \in V) \leq(1-\mu)^{n}$. However, in this case $V$ has very low combinatorial dimension and Lemma 2.3 already gives an exponential gain.

Even when $(1-\mu)^{n}<\mathbf{P}(X \in V) \leq 1$, it turns out that it is still not particularly easy to obtain (10), but one can obtain an acceptable substitute for this estimate by only replacing some of the $X_{j}$ by $Y_{j}$. Specifically, one can try to obtain an estimate roughly of the form

$$
\mathbf{P}\left(X_{1}, \ldots, X_{n} \text { span } V\right) \leq c^{m} \mathbf{P}\left(Y_{1}, \ldots, Y_{m}, X_{1}, \ldots, X_{n-m} \text { span } V\right)
$$

where $m$ is equal to a suitably small multiple of $n$ (we will eventually take $m \approx$ $n / 100)$. Strictly speaking, we will also have to absorb an additional "entropy" loss of $\left(\begin{array}{l}n \\ m\end{array}\right)$ for technical reasons, though as we will be taking $c$ arbitrarily small, this loss will ultimately be irrelevant.

The above approach (with some minor modifications) was carried out rigorously in 11 to give the bound $P_{n}=O\left(.999^{n}\right)$ which has been improved slightly to $O\left(.958^{n}\right)$ in [17. There are two main reasons why the final gain in the base was relatively small. Firstly, the chosen value of $\mu$ was small (so the $n(1-\mu)^{n}$ error was sizeable), and secondly the value of $c$ obtained was relatively large (so the gain of $c^{n}$ or $c^{(1-\gamma) n}$ was relatively weak). Unfortunately, increasing $\mu$ also causes $c$ to increase, and so even after optimizing $\mu$ and $c$, one falls well short of the conjectured bound.

In this paper we partially resolve this problem as follows. To reduce all the other losses to $\left(\frac{3}{4}+2 \epsilon_{0}\right)^{n}$ for some small $\varepsilon_{0}$, we increase $\mu$ up to $1 / 4-\epsilon_{0} / 100$, at which point the arguments of Halász and [11, 17] give (9) with $c=1$. The value $1 / 4$ for $\mu$ is optimal as it is the largest number satisfying the pointwise inequality

$$
|\cos (x)| \leq(1-\mu)+\mu \cos (2 x) \text { for all } x \in \mathbf{R},
$$

which is the Fourier-analytic analogue of (9) (with $c=1$ ). At first glance, the fact that $c=1$ seems to remove any utility to (9), as the above argument relied on obtaining gains of the form $c^{n}$ or $c^{(1-\gamma) n}$. However, we can proceed further by subdividing the collection of hyperplanes $\operatorname{Gr}\left(d_{ \pm}\right)$into two classes, namely the unexceptional spaces $V$ for which

$$
\mathbf{P}(X \in V)<\varepsilon_{1} \mathbf{P}(Y \in V)
$$

for some small constant $0<\varepsilon_{1} \ll 1$ to be chosen later (it will be much smaller than $\left.\varepsilon_{0}\right)$, and the exceptional spaces for which

$$
\varepsilon_{1} \mathbf{P}(Y \in V) \leq \mathbf{P}(X \in V) \leq \mathbf{P}(Y \in V) .
$$

The contribution of the unexceptional spaces can be dealt with by the preceding arguments to obtain a very small contribution (at most $\delta^{n}$ for any fixed $\delta>0$ given that we set $\varepsilon_{1}=\varepsilon_{1}(\gamma, \delta)$ suitably small), so it remains to consider the exceptional spaces $V$. The key new observation (which uses the Fourier-analytic methods of Halász and [11]) is that the condition (12) can be viewed as a statement that the "spectrum" of $V$ (which we will define later) has "small doubling constant". Once one sees this, one can apply theorems from inverse additive number theory (in particular a variant Freiman's theorem) to obtain some strong structural control 
on $V$ (indeed, we obtain what is essentially a complete description of the exceptional spaces $V$, up to constants depending on $\varepsilon_{0}$ and $\varepsilon_{1}$ ). This then allows us to obtain a fairly accurate bound for the total number of exceptional spaces $V$, which can then be used to estimate their contribution to (3) satisfactorily.

\section{Reduction to EXCEPTIONAL SPACES}

We now rigorously carry out the strategy outlined in Section 3. We first pick the parameter $\mu$ as

$$
\mu:=\frac{1}{4}-\frac{\varepsilon_{0}}{100}
$$

and let $Y \in\{-1,0,1\}^{n} \subset F^{n}$ be the random variables defined in Section 3 using this value of $\mu$. We let $Y_{1}, \ldots, Y_{n}$ be i.i.d. samples of $Y$, independent of the previous samples $X_{1}, \ldots, X_{n}$ of $X$. Thus we may write

$$
X=\sum_{j=1}^{n} \eta_{j}^{(1)} e_{j} ; \quad Y=\sum_{j=1}^{n} \eta_{j}^{(\mu)} e_{j}
$$

where the $\eta_{j}^{(\mu)}$ are i.i.d. samples of the random variable $\eta^{(\mu)}$ introduced in the previous section, $\eta_{j}^{(1)}$ are i.i.d. random signs, and $e_{1}, \ldots, e_{n}$ is the standard basis of $F^{n}$.

Next, we introduce a small parameter $\epsilon_{1}>0$ (which will be much smaller than $\left.\epsilon_{0}\right)$. Let us call a space $V \in \operatorname{Gr}\left(d_{ \pm}\right)$exceptional if

$$
\mathbf{P}(X \in V) \geq \varepsilon_{1} \mathbf{P}(Y \in V)
$$

and unexceptional if

$$
\mathbf{P}(X \in V)<\varepsilon_{1} \mathbf{P}(Y \in V) .
$$

As the terminology suggests, the exceptional spaces will turn out to be relatively rare compared to the unexceptional spaces, as will be seen by comparing Lemma 4.1 and Lemma 4.2 below.

Proposition 2.5 is now a consequence of the following two sublemmas.

Lemma 4.1 (Unexceptional space estimate). We have

$$
\sum_{V \in \operatorname{Gr}\left(d_{ \pm}\right): V \text { unexceptional }} \mathbf{P}\left(A_{V}\right) \leq 2^{o(n)} 2^{n} \varepsilon_{1}^{\varepsilon_{0} n / 100}
$$

where the decay rate in the o $(n)$ term can depend on $\epsilon_{0}, \epsilon_{1}$.

Lemma 4.2 (Exceptional space estimate). We have

$$
\sum_{V \in \operatorname{Gr}\left(d_{ \pm}\right): V \text { exceptional }} \mathbf{P}\left(A_{V}\right) \leq n^{-\frac{n}{2}+o(n)}
$$

where the decay rate in the $o(n)$ term can depend on $\epsilon_{0}, \epsilon_{1}$.

Indeed, upon combining Lemma 4.1 and Lemma 4.2, we obtain

$$
\sum_{V \in \operatorname{Gr}\left(d_{ \pm}\right)} \mathbf{P}\left(A_{V}\right) \leq 2^{o(n)} 2^{n} \varepsilon_{1}^{\varepsilon_{0} n / 100}+n^{-n / 2+o(n)},
$$

which implies Proposition 2.5 since $\varepsilon_{1}$ can be chosen arbitrarily small.

It remains to prove Lemma 4.1 and Lemma 4.2. We prove Lemma 4.1, which is simpler, in this section, and we leave the more difficult Lemma 4.2 to later sections. 
Proof of Lemma 4.1. We adapt some arguments form [11, 17]; the estimates here are somewhat cruder (and simpler) than in those papers, because we have the additional factor of $\varepsilon_{1}$ which can absorb several losses which can arise in this argument. We begin with a lemma.

Lemma 4.3 (Weighted Odlyzko lemma [11]). Let $0 \leq d \leq n$. If $W$ is any $d$ dimensional subspace of $F^{n}$, then

$$
\mathbf{P}(Y \in W) \leq(1-\mu)^{n-d} .
$$

Proof. Write $Y=\left(\eta_{1}, \ldots, \eta_{n}\right)$. Then there exists some $d$-tuple $\left(j_{1}, \ldots, j_{d}\right)$ of coordinates which determine the element of $W$; this means that once $\eta_{j_{1}}, \ldots, \eta_{j_{d}}$ are selected, there is only one possible choice for each of the remaining $n-d$ independent random variables $\eta_{i}$ if one wishes $Y$ to lie in $W$. Since any given value is attained by an $\eta_{i}$ with probability at most $1-\mu$, the claim follows.

Let $m$ be the nearest integer to $\varepsilon_{0} n / 100$. In addition to the random variables $X_{1}, \ldots, X_{n}$, we create more random variables $Y_{1}, \ldots, Y_{m}$ which have the same distribution as $Y$ and which are independent of each other and of $X_{1}, \ldots, X_{n}$.

Using Lemma 4.3, we have

Lemma 4.4. Let $V \in \operatorname{Gr}\left(d_{ \pm}\right)$be unexceptional, and let $B_{V, m}$ denote the event that $Y_{1}, \ldots, Y_{m}$ are linearly independent and lie in $V$. Then

$$
\mathbf{P}\left(B_{V, m}\right) \geq 2^{-o(n)}\left(2^{d_{ \pm}-n} / \varepsilon_{1}\right)^{m} .
$$

Proof. Using Bayes' identity, we can factorize

$$
\mathbf{P}\left(B_{V, m}\right)=\prod_{i=1}^{m} \mathbf{P}\left(B_{V, i} \mid B_{V, i-1}\right)
$$

where $B_{V, i}$ is the event that $Y_{1}, \ldots, Y_{i}$ are linearly independent and lie in $V$. Let $W_{i}$ be the linear subspace of $F^{n}$ spanned by $Y_{1}, \ldots, Y_{i}$. Then conditioning on any fixed value of $Y_{1}, \ldots, Y_{i-1}$ in the event $B_{V, i-1}$, we have

$$
\mathbf{P}\left(B_{V, i} \mid B_{V, i-1}\right)=\mathbf{P}(Y \in V)-\mathbf{P}\left(Y \in W_{i}\right),
$$

since $Y_{i}$ is independent of $Y_{1}, \ldots, Y_{i-1}$ and has the same distribution as $i$. By (16), (41) we have

$$
\mathbf{P}(Y \in V) \geq \frac{1}{\varepsilon_{1}} \mathbf{P}(X \in V) \geq \frac{1}{\varepsilon_{1}} 2^{-1 / n} 2^{d_{ \pm}-n} .
$$

On the other hand, since $W_{i}$ has dimension at most $m$, we see from Lemma 4.3 . (13) that

$$
\mathbf{P}\left(Y \in W_{i}\right) \leq(1-\mu)^{n-m}=\left(\frac{3}{4}+\frac{\varepsilon_{0}}{100}\right)^{n-m} .
$$

Since $m=\varepsilon_{0} n / 100+O(1)$, and $\left(\frac{3}{4}+2 \epsilon_{0}\right)^{n}<2^{d_{ \pm}-n}$ by hypothesis, we thus see that

$$
\mathbf{P}\left(Y \in W_{i}\right)=O(1 / n) \mathbf{P}(Y \in V)
$$

(for instance) if $n$ is sufficiently large depending on $\varepsilon_{0}$. Thus we have

$$
\mathbf{P}\left(B_{V, i} \mid B_{V, i-1}\right) \geq(1-O(1 / n)) 2^{d_{ \pm}-n} / \varepsilon_{1} .
$$

Multiplying this together for all $1 \leq i \leq m$, the claim follows. 
To apply this lemma, observe that $A_{V}$ and $B_{V, m}$ are clearly independent, as they involve independent sets of random variables. Thus we have

$$
\mathbf{P}\left(A_{V}\right) \leq 2^{o(n)}\left(2^{d_{ \pm}-n} / \varepsilon_{1}\right)^{-m} \mathbf{P}\left(A_{V} \wedge B_{V}\right)
$$

Now let $X_{1}, \ldots, X_{n}, Y_{1}, \ldots, Y_{m}$ be in the event $A_{V} \wedge B_{V}$. Since $Y_{1}, \ldots, Y_{m}$ are linearly independent in $V$ and $X_{1}, \ldots, X_{n}$ span $V$, we see that there exist $n-m$ vectors in $X_{1}, \ldots, X_{n}$ which, together with $Y_{1}, \ldots, Y_{m}$, span $V$. The number of possibilities for such vectors is $\left(\begin{array}{c}n \\ n-m\end{array}\right)$, which we can crudely bound by $2^{n}$. By symmetry we thus have

$$
\mathbf{P}\left(A_{V}\right) \leq 2^{o(n)}\left(2^{d_{ \pm}-n} / \varepsilon_{1}\right)^{-m} 2^{n} \mathbf{P}\left(C_{V}\right),
$$

where $C_{V}$ is the event that $Y_{1}, \ldots, Y_{m}, X_{1}, \ldots, X_{n-m}$ span $V$ and that $X_{n-m+1}, \ldots$, $X_{n}$ lie in $V$. By independence we then have

$$
\mathbf{P}\left(A_{V}\right) \leq 2^{o(n)}\left(2^{d_{ \pm}-n} / \varepsilon_{1}\right)^{-m} 2^{n} \mathbf{P}\left(Y_{1}, \ldots, Y_{m}, X_{1}, \ldots, X_{n-m} \operatorname{span} V\right) \mathbf{P}(X \in V)^{m} .
$$

Applying (4) we conclude

$$
\mathbf{P}\left(A_{V}\right) \leq 2^{o(n)}\left(2^{d_{ \pm}-n} / \varepsilon_{1}\right)^{-m} 2^{n} \mathbf{P}\left(Y_{1}, \ldots, Y_{m}, X_{1}, \ldots, X_{n-m} \operatorname{span} V\right)\left(2^{d_{ \pm}-n}\right)^{m} .
$$

Summing this over $V$ and observing that each collection of vectors $Y_{1}, \ldots$, $Y_{m}, X_{1}, \ldots, X_{n-m}$ can only span a single space $V$, we conclude

$$
\sum_{V \in \operatorname{Gr}\left(d_{ \pm}\right): V \text { unexceptional }} \mathbf{P}\left(A_{V}\right) \leq 2^{o(n)}\left(2^{d_{ \pm}-n} / \varepsilon_{1}\right)^{-m} 2^{n}\left(2^{d_{ \pm}-n}\right)^{m}
$$

and the claim follows from the choice of $m$.

\section{Structure of exceptional subspaces}

To conclude the proof of Theorem 1.2 , the only remaining task is to prove Lemma 4.2. Observe from (4) that

$$
\mathbf{P}\left(A_{V}\right) \leq \mathbf{P}(X \in V)^{n} \leq 2^{-n\left(n-d_{ \pm}\right)}
$$

for all $V \in \operatorname{Gr}\left(d_{ \pm}\right)$. Thus it will suffice to show that

$$
\mid\left\{V \in \operatorname{Gr}\left(d_{ \pm}\right): V \text { exceptional }\right\} \mid \leq n^{-\frac{n}{2}+o(n)} 2^{n\left(n-d_{ \pm}\right)} .
$$

To do this, we require a certain structural theorem about exceptional subspaces, which is the main novelty in our argument and the one which requires tools from inverse additive number theory such as Freiman's theorem.

We need some notation before we can describe the structure theorem.

Definition 5.1 (Progressions). Let $M_{1}, \ldots, M_{r}$ be integers and let $a, v_{1}, \ldots, v_{r}$ be non-zero elements of $F$. The set

$$
P:=\left\{a+m_{1} v_{1}+\ldots+m_{r} v_{r}:-M_{j} / 2<m_{j}<M_{j} / 2 \text { for all } 1 \leq j \leq r\right\}
$$

is called a generalized arithmetic progression of rank $r$; we say that the progression is symmetric if $a=0$. We say that $P$ is proper if the map

$$
\left(m_{1}, \ldots, m_{r}\right) \mapsto m_{1} v_{1}+\ldots+m_{r} v_{r}
$$

is injective when $-M_{j} / 2<m_{j}<M_{j} / 2$. If $P$ is proper and symmetric, we define the $P$-norm $\|v\|_{P}$ of a point $v=m_{1} v_{1}+\ldots+m_{r} v_{r}$ in $P$ by the formula

$$
\left\|m_{1} v_{1}+\ldots+m_{r} v_{r}\right\|_{P}:=\left(\sum_{i=1}^{r}\left(\frac{\left|m_{i}\right|}{M_{i}}\right)^{2}\right)^{1 / 2}
$$


Let $V \in \operatorname{Gr}\left(d_{ \pm}\right)$be an exceptional space, with a representation of the form

$$
V=\left\{\left(x_{1}, \ldots, x_{n}\right) \in F^{n}: x_{1} a_{1}+\ldots+x_{n} a_{n}=0\right\}
$$

for some elements $a_{1}, \ldots, a_{n} \in F$. We shall refer to $a_{1}, \ldots, a_{n}$ as the defining coordinates for $V$.

Theorem 5.2 (Structure theorem). There is a constant $C=C\left(\epsilon_{0}, \epsilon_{1}\right)$ such that the following holds. Let $V$ be an exceptional hyperplane in $\operatorname{Gr}\left(d_{ \pm}\right)$and let $a_{1}, \ldots, a_{n}$ be its defining coordinates. Then there exist integers

$$
1 \leq r \leq C
$$

and $M_{1}, \ldots, M_{r} \geq 1$ with the volume bound

$$
M_{1} \ldots M_{r} \leq C 2^{n-d_{ \pm}}
$$

and non-zero elements $v_{1}, \ldots, v_{r} \in F$ such that the following hold.

- (i) (Defining coordinates lie in a progression) The symmetric generalized arithmetic progression

$$
P:=\left\{m_{1} v_{1}+\ldots+m_{r} v_{r}:-M_{j} / 2<m_{j}<M_{j} / 2 \text { for all } 1 \leq j \leq r\right\}
$$

is proper and contains all the $a_{i}$.

- (ii) (Bounded norm) The $a_{i}$ have small P-norm:

$$
\sum_{j=1}^{n}\left\|a_{j}\right\|_{P}^{2} \leq C .
$$

- (iii) (Rational commensurability) The set $\left\{v_{1}, \ldots, v_{r}\right\} \cup\left\{a_{1}, \ldots, a_{n}\right\}$ is contained in the set

$$
\left\{\frac{p}{q} v_{1}: p, q \in \mathbf{Z} ; q \neq 0 ;|p|,|q| \leq n^{o(n)}\right\} .
$$

Remark 5.3. The condition (i) asserts that the defining coordinates $a_{1}, \ldots, a_{n}$ of $V$ lie in a fairly small generalized arithmetic progression with bounded rank $O(1)$. The condition (23) asserts, furthermore, that this progression is contained in a (rank one) arithmetic progression of length $n^{o(n)}$. Thus we have placed $\left\{a_{1}, \ldots, a_{n}\right\}$ inside two progressions, one of small size but moderately large rank and one of rank one but of fairly huge size (but the dimensions are still smaller than $n^{n / 2}$ or $|F|$ ).

Remark 5.4. The structure theorem is fairly efficient; if $a_{1}, \ldots, a_{n}$ obey the conclusions of the theorem, then from (22) and the theory of random walks (taking advantage of the boundedness of $r$, which will play the role of dimension) we expect the random variables $\eta_{1}^{(1)} a_{1}+\ldots+\eta_{1}^{(1)} a_{n}$ and $\eta_{1}^{(\mu)} a_{1}+\ldots+\eta_{1}^{(\mu)} a_{n}$ to be distributed fairly uniformly on $P$ and to decay rapidly away from $P$, and so we expect the probabilities $\mathbf{P}(X \in V)$ and $\mathbf{P}(Y \in V)$ to be comparable (up to a constant).

We shall prove the structure theorem in later sections. For the remainder of this section, we show how the structure theorem can be used to prove Lemma 4.2. In the sequel all of the $o\left(\right.$ ) factors are allowed to depend on $\epsilon_{0}, \epsilon_{1}$.

Let $V \in \operatorname{Gr}\left(d_{ \pm}\right)$be an exceptional hyperplane. Then $V$ has $|F|-1$ representations of the form (19), one for each non-zero normal vector of $V$. Let us call an 
$n$-tuple $\left(a_{1}, \ldots, a_{n}\right)$ of elements in $F$ exceptional if it obeys the conclusions of the structure theorem for at least one progression $P$; thus we have

$$
\begin{aligned}
\mid\left\{V \in \operatorname{Gr}\left(d_{ \pm}\right): V \text { exceptional }\right\} \mid \\
\quad=\frac{1}{|F|-1} \mid\left\{\left(a_{1}, \ldots, a_{n}\right) \in F^{n}:\left(a_{1}, \ldots, a_{n}\right) \text { exceptional }\right\} \mid,
\end{aligned}
$$

and so it now suffices to show

$$
\mid\left\{\left(a_{1}, \ldots, a_{n}\right) \in F^{n}:\left(a_{1}, \ldots, a_{n}\right) \text { exceptional }\right\}\left|\leq n^{o(n)} n^{-n / 2} 2^{n\left(n-d_{ \pm}\right)}\right| F \mid .
$$

We now need to count the number of exceptional $\left(a_{1}, \ldots, a_{n}\right)$. We first observe that we may fix the parameter $r$ in the conclusion of the structure theorem, since the number of $r$ is at most $C=n^{o(n)}$. Similarly we may fix each $M_{1}, \ldots, M_{r}$, since the total number of choices here is at most $\left(C 2^{n-d_{ \pm}}\right)^{r}=n^{o(n)}$.

The number of possible choices for $v_{1}$ is at most $|F|$. Once this vector is selected, we see from (23) that there are at most $n^{o(n)}$ possible choices for each of the remaining $r-1=O(1)$ vectors. Putting the estimates together, we can conclude that the total number of possible vectors $\left(v_{1}, \ldots, v_{r}\right)$ which could be chosen is at most $n^{o(n)}|F|$.

It now suffices to show that, for each fixed choice of $r, M_{1}, \ldots, M_{r}, v_{1}, \ldots, v_{r}$ (which in particular fixes $P$ ), the number of possible exceptional $\left(a_{1}, \ldots, a_{n}\right)$ is at most $n^{o(n)} n^{-n / 2} 2^{n\left(n-d_{ \pm}\right)}$. We are going to use the bound on the $P$-norms of the $a_{i}$. Our task is to show that for any constant $C$

$$
\left|\left\{\left(a_{1}, \ldots, a_{n}\right) \in P^{n}: \sum_{j=1}^{n}\left\|a_{j}\right\|_{P}^{2} \leq C\right\}\right| \leq n^{o(n)} n^{-n / 2} 2^{n\left(n-d_{ \pm}\right)} .
$$

We shall use Gaussian-type methods. To start, notice that

$$
\left|\left\{\left(a_{1}, \ldots, a_{n}\right) \in P^{n}: \sum_{j=1}^{n}\left\|a_{j}\right\|_{P}^{2} \leq C\right\}\right| \leq n^{o(n)} \sum_{\left(a_{1}, \ldots, a_{n}\right) \in P^{n}} \exp \left(-n \sum_{j=1}^{n}\left\|a_{j}\right\|_{P}^{2}\right) .
$$

The right hand side can be rewritten as

$$
n^{o(n)}\left(\sum_{a \in P} \exp \left(-n\|a\|_{P}^{2}\right)\right)^{n}=n^{o(n)}\left(\prod_{j=1}^{r} \sum_{\left|m_{j}\right| \leq M_{j}} e^{-n m_{j}^{2} / M_{j}^{2}}\right)^{n} .
$$

Using the elementary bound

$$
\sum_{|m| \leq M} e^{-n m^{2} / M^{2}}=O\left(1+M n^{-1 / 2}\right)
$$

we can bound the right hand side from above by

$$
n^{o(n)}\left(\prod_{1 \leq j \leq r}\left(1+\frac{M_{j}}{\sqrt{n}}\right)\right)^{n}
$$

Since $r=O(1)$ and $M_{1}, \ldots, M_{r} \geq 1$, the product $\prod_{1 \leq j \leq r}\left(1+\frac{M_{j}}{\sqrt{n}}\right)$ can be bounded (rather crudely) by $O\left(1+n^{-1 / 2} M_{1} \ldots M_{r}\right)$. This leads us to the bound

$$
n^{o(n)}\left(1+n^{-1 / 2} M_{1} \ldots M_{r}\right)^{n} \text {. }
$$

Now we use the information (see (21) in the Structure Theorem) that $M_{1} \ldots M_{r}$ is at most $C 2^{n-d_{ \pm}}$. The bound becomes

$$
n^{o(n)}\left(1+n^{-1 / 2} C 2^{n-d_{ \pm}}\right)^{n}=n^{-n / 2+o(n)} 2^{n\left(n-d_{ \pm}\right)}
$$


as desired, thanks to the hypothesis $2^{d_{ \pm}-n} \leq 100 / \sqrt{n}$.

This concludes the proof of Theorem 1.2 except for the Structure Theorem, which we turn to next. This proof requires a heavy use of tools from additive combinatorics. We are going to introduce these tools in the next section.

\section{GenerAlized ARIthmetic PROGRESSIONS AND INVERSE THEOREMS}

In this section we review some notation and results from additive combinatorics and briefly sketch how they will be used to prove Theorem 5.2. The rigorous proof of this theorem will begin in Section 7

Let $A$ and $B$ be finite subsets of $G$. By $A+B:=\{a+b: a \in A, b \in B\}$ we denote the set of all elements of $G$ which can be represented as the sum of an element from $A$ and an element from $B ; A-B:=\{a-b: a \in A, b \in B\}$ is defined similarly. Moreover, for any positive integer $l$, we recursively define $l A:=(l-1) A+A$. Thus $l A$ is the set of $l$-fold sums in $A$ (allowing repetition).

The doubling constant of a finite non-empty set $A \subseteq F$ is defined to be the ratio $|A+A| /|A|$. It is easy to see that if $A$ is a dense subset (with constant density $\delta$ ) of a proper generalized arithmetic progression of constant rank $r$, then the doubling number of $A$ is bounded from above by a constant depending on $\delta$ and $r$ (in fact it is bounded by $\delta^{-1} 2^{r}$ ).

In the mid 1970s, Freiman 6 proved a remarkable result in the converse direction, which asserts that being a dense subset of a proper arithmetic progression of constant rank $r$ is the only reason for a finite set $A$ of integers to have small doubling constant:

Theorem 6.1 (Freiman's theorem [6]). For any constant $C$ there are constants $r$ and $\delta$ such that the following holds. For any finite set $A$ of integers such that $|A+A| \leq C|A|$, there is a proper arithmetic progression $P$ of rank $r$ such that $A \subseteq P$ and $|A| /|P| \geq \delta$.

Freiman's proof was rather complex. A cleaner proof, based on Freiman's ideas, is presented by Bilu in [1. Ruzsa [15] gave a different and quite short proof; this was then refined by Chang 4]. There are explicit bounds known on the values of $r$ and $\delta$ in terms of $C$, but for our application these bounds will only influence the $o(1)$ term in our final result and so we will not keep track of them here.

Freiman's theorem was initially phrased in the integers $\mathbf{Z}$, but it can easily be transferred to a finite field $F$ of prime order if $F$ is sufficiently large:

Theorem 6.2. For any constant $C$ there are constants $r$ and $\delta$ such that the following holds. Let $F$ be a finite field of prime order, and let $A$ be a non-empty subset of $F$ such that $|A+A| \leq C|A|$. Then, if $|F|$ is sufficiently large depending on $|A|$, there is a generalized arithmetic progression $P$ of rank $r$ such that $A \subset P$ and $|A| /|P| \geq \delta$.

This theorem follows from Freiman's original theorem and "rectification" theorems such as [2, Theorem 3.1] (see also [7) to map $A$ via a Freiman isomorphism to the integers $\mathbf{Z}$ (using the hypothesis that $F$ is large depending on $A$; $|F| \geq \exp (C|A|)$ would do). It is also a special case of the version of Freiman's theorem established in 8 for an arbitrary abelian group. In fact, that result allows one to remove the hypothesis that $|F|$ is sufficiently large depending on $|A|$. 
A generalized arithmetic progression is not always proper. We can, however, make this assumption whenever we like (at the cost of a constant factor) thanks to the following lemma.

Lemma 6.3 (Progressions lie inside proper progressions). There is a constant $C$ such that the following holds. Let $P$ be a generalized arithmetic progression of rank $r$ in an abelian group $G$. Suppose that every non-zero element of $G$ has order at least $r^{C r^{3}}|P|$. Then there exists a proper arithmetic progression $Q$ of rank at most $r$ containing $P$ and

$$
|Q| \leq r^{C r^{3}}|P|
$$

The proof of this lemma arises purely from the geometry of numbers (in particular, Minkowski's second theorem) and is independent of the rest of this paper. In order not to distract the reader, we defer this rather technical proof to the Appendix.

Finally, we are going to need the following lemma, based on a covering argument of Ruzsa [16. We say that a set $A$ is symmetric if the set $-A:=\{-a, a \in A\}$ is equal to $A$.

Lemma 6.4 (Sumset estimates). Let $A$ be a symmetric finite subset of an abelian group $G$ such that $|4 A| \leq C|A|$ for some $C \geq 1$. Then for any $k \geq 4$

$$
|k A| \leq\left(\begin{array}{c}
C+k-3 \\
k-2
\end{array}\right) C|A| .
$$

Proof. We can assume that $k \geq 4$. Consider the sets $\eta+A$ as $\eta$ ranges inside $3 A$. Each set has cardinality $|A|$ and is contained inside $4 A$. Thus we may find a maximal disjoint collection $\{\xi+A: \eta \in X\}$ where $X \subseteq 3 A$ has cardinality $|X| \leq|4 A| /|A| \leq C$. Then for any $\xi \in 3 A$ there exists $\eta \in X$ such that $\eta+\Lambda$ intersects $\xi+A$; otherwise this would contradict maximality. But this implies that $\xi \in X+A-A=2 A+X$. Thus we have

$$
A+2 A=3 A \subseteq 2 A+X .
$$

Iterating this, we obtain

$$
k A \subset 2 A+(k-2) X
$$

for all $k \geq 2$. Thus

$$
|k A| \leq|2 A||(k-2) X| \leq C|A|(k-2) X \mid .
$$

To conclude the proof, notice that for any $l$

$$
|l X| \leq\left(\begin{array}{c}
|X|+l-1 \\
l
\end{array}\right) .
$$

In fact one can replace the condition $|4 A| \leq C|A|$ by a weaker condition $|3 A| \leq$ $C|A|$.

We are going to use Theorem 6.2 to derive a structural property of the defining vectors $\left\{a_{1}, \ldots, a_{n}\right\}$ of $V$ in the Structure Theorem. To be more precise, we are going to apply Theorem 6.2 to a set $A$ with small doubling constant which contains the majority of the defining vectors $\left\{a_{1}, \ldots, a_{n}\right\}$. Thus we obtain a generalized arithmetic progression $P$ containing $A$ (and with it most of the $a_{i}$ ). It will be easy to extend $P$ to contain all $a_{i}$ without violating the properties we care about. 
The application of Theorem 6.2 is not immediate. In the next section, we shall provide a Fourier-analytic argument which leads to the definition of $A$. The starting point of this argument is based on the method in [11, which is motivated by an observation of Halász [9]; this method shall be the focus of the next section.

\section{HalÁsZ-TYPe ARGUMENTS}

Let $V \in \operatorname{Gr}\left(d_{ \pm}\right)$be an exceptional space with the representation (19). In order to obtain the desired structural control on the defining coordinates $a_{1}, \ldots, a_{n}$, we shall first use Fourier analysis to gain some strong control on the "spectrum" $\Lambda$ of $a_{1}, \ldots, a_{n}$ (which we will define in (28) ) and then apply the inverse Fourier transform to recover information about $a_{1}, \ldots, a_{n}$.

We turn to the details. By Fourier expansion we have

$$
1_{\left(x_{1}, \ldots, x_{n}\right) \in V}=\frac{1}{|F|} \sum_{\xi \in F} e_{p}\left(x_{1} a_{1} \xi+\ldots+x_{n} a_{n} \xi\right)
$$

for all $\left(x_{1}, \ldots, x_{n}\right) \in F^{n}$, where $p=|F|$ and $e_{p}$ is the primitive character $e_{p}(x):=$ $e^{2 \pi i x / p}$. By (14) we thus have (by linearity of expectation and by independence)

$$
\begin{aligned}
\mathbf{P}(X \in V) & =\mathbf{E}\left(1_{X \in V}\right) \\
& =\frac{1}{|F|} \sum_{\xi \in F} \mathbf{E}\left(e_{p}\left(\eta_{1}^{(1)} a_{1} \xi+\ldots+\eta_{n}^{(1)} a_{n} \xi\right)\right) \\
& =\frac{1}{|F|} \sum_{\xi \in F} \prod_{j=1}^{n} \mathbf{E}\left(e_{p}\left(\eta_{j}^{(1)} a_{j} \xi\right)\right) \\
& =\frac{1}{|F|} \sum_{\xi \in F} \prod_{j=1}^{n} \cos \left(2 \pi a_{j} \xi / p\right) .
\end{aligned}
$$

In particular we have

$$
\begin{aligned}
\mathbf{P}(X \in V) & \leq \frac{1}{|F|} \sum_{\xi \in F} \prod_{j=1}^{n}\left|\cos \left(2 \pi a_{j} \xi / p\right)\right| \\
& =\frac{1}{|F|} \sum_{\xi \in F} \prod_{j=1}^{n}\left|\cos \left(\pi a_{j} \xi / p\right)\right| \\
& =\frac{1}{|F|} \sum_{\xi \in F} \prod_{j=1}^{n}\left(\frac{1}{2}+\frac{1}{2} \cos \left(2 \pi a_{j} \xi / p\right)\right)^{1 / 2}
\end{aligned}
$$

where the first identity follows from the substitution $\xi \mapsto \xi / 2$ on $F$, noting that the quantity $\left|\cos \left(\pi a_{j} \xi / p\right)\right|$ is still periodic in $\xi$ with period $p$. Arguing similarly with $Y$, we have

$$
\begin{aligned}
\mathbf{P}(Y \in V) & =\frac{1}{|F|} \sum_{\xi \in F} \prod_{j=1}^{n} \mathbf{E}\left(e_{p}\left(\eta_{j}^{(\mu)} a_{j} \xi\right)\right) \\
& =\frac{1}{|F|} \sum_{\xi \in F} \prod_{j=1}^{n}\left((1-\mu)+\mu \cos \left(2 \pi a_{j} \xi / p\right)\right) .
\end{aligned}
$$


To summarize, if we introduce the non-negative quantities

(25) $f(\xi):=\prod_{j=1}^{n}\left(\frac{1}{2}+\frac{1}{2} \cos \left(2 \pi a_{j} \xi / p\right)\right)^{1 / 2}, \quad g(\xi):=\prod_{j=1}^{n}\left((1-\mu)+\mu \cos \left(2 \pi a_{j} \xi / p\right)\right)$,

then we have

$$
\mathbf{P}(X \in V) \leq \frac{1}{|F|} \sum_{\xi \in F} f(\xi), \quad \mathbf{P}(Y \in V)=\frac{1}{|F|} \sum_{\xi \in F} g(\xi) .
$$

We now give a crucial comparison estimate between $f$ and $g$.

Lemma 7.1. For all $\xi \in F$, we have $f(\xi) \leq g(\xi)^{1 / 4 \mu}$.

Proof. By (25) it suffices to show that

$$
\left(\frac{1}{2}+\frac{1}{2} \cos \theta\right)^{1 / 2} \leq((1-\mu)+\mu \cos \theta)^{1 / 4 \mu}
$$

for any $\theta$. Writing $\cos \theta=1-2 x$ for some $0<x \leq 1$ (the $x=0$ case being trivial), this can be rearranged to become

$$
\frac{\log (1-x)-\log (1-0)}{x} \leq \frac{\log (1-2 \mu x)-\log (1-0)}{2 \mu x} .
$$

But this follows from the concavity of the function $\log (1-x)$ on $0 \leq x \leq 1$ and the fact (from (13)) that $0<2 \mu<1$.

Since $g(\xi)$ is clearly bounded by 1 and since $\mu<1 / 4$ by (13), it follows that for every $\xi$

$$
f(\xi) \leq g(\xi),
$$

which when combined with (26) gives

$$
\mathbf{P}(X \in V) \leq \mathbf{P}(Y \in V) .
$$

We now refine this argument to exploit the additional (and critical) hypothesis (15) that

$$
\mathbf{P}(X \in V) \geq \varepsilon_{1} \mathbf{P}(Y \in V) .
$$

Let $\epsilon_{2}>0$ be a sufficiently small positive constant (compared to $\epsilon_{1}$ ). Define the spectrum $\Lambda \subseteq F$ of $\left\{a_{1}, \ldots, a_{n}\right\}$ to be the set

$$
\Lambda:=\left\{\xi \in F: f(\xi) \geq \varepsilon_{2}\right\} .
$$

Note that $\Lambda$ is symmetric around the origin: $\Lambda=-\Lambda$. Next we make the elementary observation that

$$
1-100\|x\|^{2} \leq \cos (2 \pi x) \leq 1-\frac{1}{100}\|x\|^{2}
$$

(say), where $\|x\|$ is the distance of $x$ to the nearest integer. From (25) we thus have (with room to spare)

$$
f(\xi) \leq \exp \left(-\frac{1}{1000} \sum_{j=1}^{n}\left\|a_{j} \cdot \xi / p\right\|^{2}\right)
$$


and hence there is a constant $C\left(\epsilon_{2}\right)$ depending on $\epsilon_{2}$ such that

$$
\left(\sum_{j=1}^{n}\left\|a_{j} \cdot \xi / p\right\|^{2}\right)^{1 / 2} \leq C\left(\varepsilon_{2}\right)
$$

for all $\xi \in \Lambda$.

We now obtain some cardinality bounds on $\Lambda$ and of the iterated sumsets $k \Lambda$.

Lemma 7.2. There is a constant $C$ depending on $\epsilon_{0}, \epsilon_{1}, \epsilon_{2}$ such that

$$
C^{-1} 2^{-\left(n-d_{ \pm}\right)}|F| \leq|\Lambda| \leq C 2^{-\left(n-d_{ \pm}\right)}|F| .
$$

Furthermore, for every integer $k \geq 4$

$$
|k \Lambda| \leq\left(\begin{array}{c}
C+k-3 \\
k-2
\end{array}\right) C 2^{-\left(n-d_{ \pm}\right)}|F| .
$$

Remark 7.3. The lemma implies that $\Lambda$ has a small doubling constant. At this point one could apply Theorem 6.2 to gain further control on $\Lambda$. While this can be done, it is more convenient for us to apply the inverse Fourier transform and work on a certain "dual" set to $\Lambda$, which then contains most of the $a_{j}$. A key point in (32), which we will exploit in the proof of Lemma 7.4 below, is that the growth of constants is only polynomial in $k$ rather than exponential.

Since $\epsilon_{i}$ depends on $\epsilon_{0}, \ldots, \epsilon_{i-1}$, it would suffice to say that $C$ depends only on $\epsilon_{0}$.

Proof. From (15), (26) we have

$$
\frac{1}{|F|} \sum_{\xi \in F} f(\xi) \geq \varepsilon_{1} \frac{1}{|F|} \sum_{\xi \in F} g(\xi)
$$

But from Lemma 7.1. the definition of $\Lambda$, and the crucial fact that $\mu<1 / 4$, we know that

$$
\begin{aligned}
\frac{1}{|F|} \sum_{\xi \notin \Lambda} f(\xi) & \leq \varepsilon_{2}^{1-4 \mu} \frac{1}{|F|} \sum_{\xi \notin \Lambda} f^{4 \mu}(\xi) \\
& \leq \varepsilon_{2}^{1-4 \mu} \frac{1}{|F|} \sum_{\xi \in F} g(\xi) .
\end{aligned}
$$

Now we are going to make an essential use of the assumption that $\mu$ is less than $1 / 4$. Given this assumption, we can guarantee that the contribution of $\xi$ outside the spectrum is negligible, by choosing $\varepsilon_{2}$ sufficiently small compared to $\varepsilon_{1}$. This results in the equalities

$$
\sum_{\xi \in \Lambda} f(\xi)=\Theta\left(\sum_{\xi \in F} f(\xi)\right)=\Theta\left(\sum_{\xi \in F} g(\xi)\right)=\Theta(|F| \mathbf{P}(X \in V))=\Theta\left(2^{d_{ \pm}-n}|F|\right),
$$

where the constants in the $\Theta()$ notation depend on $\epsilon_{0}, \epsilon_{1}, \epsilon_{2}$. The bounds on $|\Lambda|$ now follow directly from the fact that for any $\xi \in \Lambda, \epsilon_{2} \leq f(\xi) \leq 1$.

It remains to prove (32). In view of Lemma 6.4 it suffices to show that there is a constant $C$ such that

$$
|4 \Lambda| \leq C|\Lambda|
$$


Notice that if $\xi \in 4 \Lambda$, then by (30) and the triangle inequality we have

$$
\left(\sum_{j=1}^{n}\left\|a_{j} \cdot \xi / p\right\|^{2}\right)^{1 / 2} \leq C\left(\varepsilon_{2}\right)
$$

for some constant $C\left(\epsilon_{2}\right)$ depending on $\epsilon_{2}$ (we abuse the notation a little bit, as the two $C\left(\epsilon_{2}\right)$ are not necessarily the same). From (29), (25) we conclude a bound of the form

$$
f(\xi) \geq c\left(\varepsilon_{2}\right)>0
$$

Thus

$$
|4 \Lambda| \leq c\left(\varepsilon_{2}\right)^{-1} \sum_{\xi \in G} f(\xi) \leq c\left(\varepsilon_{2}\right)^{-1} \sum_{\xi \in F} g(\xi)=\Theta\left(2^{d_{ \pm}-n}|F|\right)=\Theta(|\Lambda|),
$$

concluding the proof.

We now pass from control of the spectrum back to control on $\left\{a_{1}, \ldots, a_{n}\right\}$, using the inverse Fourier transform. For any $x \in F$, define the norm $\|x\|_{\Lambda}$ by

$$
\|x\|_{\Lambda}:=\left(\frac{1}{|\Lambda|^{2}} \sum_{\xi, \xi^{\prime} \in \Lambda}\left\|x\left(\xi-\xi^{\prime}\right) / p\right\|^{2}\right)^{1 / 2} .
$$

It is easy to see that this is a quantity between 0 and 1 which obeys the triangle inequality $\|x+y\|_{\Lambda} \leq\|x\|_{\Lambda}+\|y\|_{\Lambda}$. From the triangle inequality again we have

$$
\begin{aligned}
\|x\|_{\Lambda} & \leq\left(\frac{1}{|\Lambda|^{2}} \sum_{\xi, \xi^{\prime} \in \Lambda}\|x \xi / p\|^{2}\right)^{1 / 2}+\left(\frac{1}{|\Lambda|^{2}} \sum_{\xi, \xi^{\prime} \in \Lambda}\left\|x \xi^{\prime} / p\right\|^{2}\right)^{1 / 2} \\
& =2\left(\frac{1}{|\Lambda|} \sum_{\xi}\|x \xi / p\|^{2}\right)^{1 / 2} .
\end{aligned}
$$

Thus by square-summing (30) for all $\xi \in \Lambda$, we obtain

$$
\sum_{j=1}^{n}\left\|a_{j}\right\|_{\Lambda}^{2} \leq C\left(\varepsilon_{2}\right)
$$

Thus we expect many of the $a_{j}$ to have small $\Lambda$ norm. On the other hand, we are going to show that the set of elements with small $\Lambda$ norm has constant doubling number, thanks to the following lemma.

Lemma 7.4. There is a constant $C$ such that the following holds. Let $A \subseteq F$ denote the "Bohr set"

$$
A:=\left|\left\{x \in F:\|x\|_{\Lambda} \leq \frac{1}{100}\right\}\right|
$$

Then we have

$$
C^{-1} 2^{n-d_{ \pm}} \leq|A| \leq|A+A| \leq C 2^{n-d_{ \pm}}
$$

Proof. Let us first establish the upper bound. We introduce the normalized Fourier transform of $\Lambda$ :

$$
h(x):=\frac{1}{|\Lambda|} \sum_{\xi \in \Lambda} e_{p}(x \xi) .
$$


If $x \in A+A$, then we have $\|x\|_{\Lambda} \leq \frac{1}{50}$ by the triangle inequality. Furthermore, using (29), we have

$$
\begin{aligned}
|h(x)|^{2} & =\Re h(x) \overline{h(x)} \\
& =\Re \frac{1}{|\Lambda|^{2}} \sum_{\xi, \xi^{\prime} \in \Lambda} e_{p}\left(x\left(\xi-\xi^{\prime}\right)\right) \\
& =\frac{1}{|\Lambda|^{2}} \sum_{\xi, \xi^{\prime} \in \Lambda} \cos \left(2 \pi x\left(\xi-\xi^{\prime}\right) / p\right) \\
& \geq \frac{1}{|\Lambda|^{2}} \sum_{\xi, \xi^{\prime} \in \Lambda} 1-100\left\|x\left(\xi-\xi^{\prime}\right) / p\right\|^{2} \\
& =1-100\|x\|_{\Lambda}^{2} .
\end{aligned}
$$

As $\|x\|_{\Lambda} \leq \frac{1}{50}$, it follows that

$$
|h(x)| \geq \sqrt{1-100(1 / 50)^{2}}>1 / 2 .
$$

On the other hand, from the Parseval identity we have

$$
\sum_{x \in F}|h(x)|^{2}=|F| /|\Lambda|
$$

combining these two estimates, we obtain

$$
|A+A| \leq 4|F| /|\Lambda|
$$

and the claim now follows from (31).

Since the bound $|A| \leq|A+A|$ is trivial, it now suffices to prove the lower bound on $A$. This proof has two parts. First we show that if $|h(x)|$ is large (close to 1 ), then $\|x\|_{\Lambda}$ is small (close to zero). Next, we prove that there are many $x$ such that $|h(x)|$ is large.

The first part is simple and similar to the above argument. Using the fact that $\|x\|_{\Lambda} \leq \frac{1}{50}$ and (29), we have

$$
\begin{aligned}
|h(x)|^{2} & =\Re h(x) \overline{h(x)} \\
& =\Re \frac{1}{|\Lambda|^{2}} \sum_{\xi, \xi^{\prime} \in \Lambda} e_{p}\left(x\left(\xi-\xi^{\prime}\right)\right) \\
& =\frac{1}{|\Lambda|^{2}} \sum_{\xi, \xi^{\prime} \in \Lambda} \cos \left(2 \pi x\left(\xi-\xi^{\prime}\right) / p\right) \\
& \leq \frac{1}{|\Lambda|^{2}} \sum_{\xi, \xi^{\prime} \in \Lambda} 1-\frac{1}{100}\left\|x\left(\xi-\xi^{\prime}\right) / p\right\|^{2} \\
& =1-\frac{1}{100}\|x\|_{\Lambda}^{2} .
\end{aligned}
$$

It follows that if $|h(x)| \geq 1-10^{-4}$, then $x \in A$.

Now we are going to show that the set of those $x$ such that $|h(x)| \geq 1-10^{-4}$ has cardinality $\Theta\left(2^{n-d_{ \pm}}\right)$. This proof is trickier and we need to adapt an argument from [7] (see also 8]), which requires the full strength of (32). Let $k$ be a large integer to be chosen later, and for each $\xi \in F$, let $r_{k}(\xi)$ be the number of representations 
of the form $\xi=\xi_{1}+\ldots+\xi_{k}$ where $\xi_{1}, \ldots, \xi_{k} \in \Lambda$. Clearly we have

$$
\sum_{\xi \in k \Lambda} r_{k}(\xi)=|\Lambda|^{k}
$$

and so by Cauchy-Schwarz

$$
\sum_{\xi \in F}\left|r_{k}(\xi)\right|^{2} \geq|\Lambda|^{2 k} /|k \Lambda|
$$

By Plancherel we have

$$
\sum_{\xi \in F}\left|r_{k}(\xi)\right|^{2}=\frac{1}{|F|} \sum_{x \in F}\left|\sum_{\xi \in F} r_{k}(\xi) e_{p}(x \xi)\right|^{2} .
$$

But we have

$$
\begin{aligned}
\left|\sum_{\xi \in F} r_{k}(\xi) e_{p}(x \xi)\right| & =\mid \sum_{\xi_{1}, \ldots, \xi_{k} \in \Lambda} e_{p}\left(x\left(\xi_{1}+\ldots+\xi_{k}\right) \mid\right. \\
& =|\Lambda|^{k}|h(x)|^{k}
\end{aligned}
$$

and hence

$$
\sum_{x \in F}|h(x)|^{2 k} \geq|F| /|k \Lambda|
$$

Combining this with (34), we obtain

$$
\sum_{x \in F:|h(x)| \geq(|\Lambda| / 2|k \Lambda|)^{1 /(2 k-2)}}|h(x)|^{2 k} \geq|F| / 2|k \Lambda| ;
$$

since $h(x)$ is trivially bounded by 1 , we thus conclude

$$
\left|\left\{x \in F:|h(x)| \geq(|\Lambda| / 2|k \Lambda|)^{1 /(2 k-2)}\right\}\right| \geq|F| / 2|k \Lambda| .
$$

Inserting the bounds (31), (32), we have

$$
(|\Lambda| / 2|k \Lambda|)^{1 /(2 k-2)} \geq\left[\left(\begin{array}{c}
C+k-3 \\
k-2
\end{array}\right) C\right]^{1 /(2 k-2)}
$$

for some constant $C$. Choosing $k$ sufficiently large (say $k=C^{2}+10^{10}$ ), we can guarantee that the right hand side is at least $1-10^{-4}$. On the other hand $k$ is still a constant, so $|k \Lambda|=O\left(2^{d_{ \pm}-n}|F|\right)$. It follows that

$$
\left|\left\{x \in F:|h(x)| \geq 1-10^{-4}\right\}\right|=\Theta\left(2^{n-d_{ \pm}}\right),
$$

proving the lower bound on $A$.

\section{Proof of the Structure Theorem}

We are now in a position to prove the Structure Theorem. In this section we allow the constants in the $O\left(\right.$ ) notation to depend on $\epsilon_{0}, \epsilon_{1}, \epsilon_{2}$. From Lemma 7.4 we already know that $A$ has doubling constant $O(1)$. Furthermore, it is easy to show that $A$ contains most of the $a_{i}$. Indeed, if $a_{i}$ is not in $A$, then its $\Lambda$-norm is at least $1 / 100$; (33) then shows that

$$
\left|\left\{1 \leq i \leq n: a_{i} \notin A\right\}\right|=O(1) .
$$

We now apply Theorem 6.2 to $A$, to conclude that there is a generalized arithmetic progression $P$ of rank $O(1)$ containing $A$ and with $|A|=\Theta(|P|)=\Theta\left(2^{n-d_{ \pm}}\right)$. By Lemma 6.3 we can assume that $P$ is proper (note that every non-zero element of $F$ has order $|F|=p \geq n^{n / 2}$, which is certainly much larger than $|A|$ ). 
This progression $P$ is close to, but not quite, what we need for the structure theorem. Thus we shall now perform a number of minor alterations to $P$ in order to make it exactly match the conclusions of the structure theorem. Each of our alterations will preserve the following properties claimed in the structure theorem, namely

$$
\begin{gathered}
\operatorname{rank}(P)=O(1) \text { and } \\
|P|=M_{1} \ldots M_{r}=O\left(2^{n-d_{ \pm}}\right),
\end{gathered}
$$

though of course the $O($ ) constants may worsen with each of the alterations.

First, we can include the elements of $\left\{a_{1}, \ldots, a_{n}\right\} \backslash A$ in $P$ simply by adding each exceptional $a_{j}$ as a new basis vector $v_{j}^{\prime}$ (with the corresponding length $M_{j}^{\prime}$ set equal to 3 ). This enlargement of $P$ (which by abuse of notation we shall continue to call $P$ ) now contains $A$ and all of the $a_{j}$ and obeys the bounds (35), (36) with slightly worse constants. Notice that by Lemma 6.3 we can always assume $P$ is proper, without affecting the validity of (35) and (36) other than in the constants.

It now remains to verify the estimate (22) on the $P$-norm, as well as the rational commensurability claim.

To verify (22), consider the sum $\sum_{j}\left\|a_{j}\right\|_{P}^{2}$. For the exceptional $a_{j}$ (which were added to $P$ afterwards) $\left\|a_{j}\right\|_{P}=O(1)$. However, the number of these $a_{j}$ is $O(1)$. For the remaining $a_{j}$, notice that $P$ contains $k a_{j}$ for all $k<\frac{1}{100\left\|a_{j}\right\|_{\Lambda}}$. Thus, (22) follows from (33) which asserts that $\sum_{j}\left\|a_{j}\right\|_{\Lambda}^{2}=O(1)$. This leaves the verification of the rational commensurability as the only remaining task.

Let us, as usual, write

$$
P=\left\{m_{1} v_{1}+\cdots+m_{r} v_{r} \mid-M_{j} / 2 \leq m_{j} \leq M_{j} / 2\right\} .
$$

We consider $P$ together with the map $\Phi: P \rightarrow \mathbf{R}^{r}$ which maps $m_{1} v_{1}+\cdots+m_{r} v_{r}$ to $\left(m_{1}, \ldots, m_{r}\right)$. Since $P$ is proper, this map is bijective. We set $U:=\left\{a_{1}, \ldots, a_{n}\right\} \subseteq$ $P$.

We know that the progression $P$ contains $U$, but we do not know yet that $U$ "spans" $P$ in the sense that the set $\Phi(U)$ has full rank (i.e., it spans $\mathbf{R}^{r}$ ). However, this is easily rectified by a rank reduction argument. Suppose that $\Phi(U)$ did not have full rank. We are going to produce a new proper generalized arithmetic progression $P^{\prime}$ which still contains $U$ and satisfies (36) but has rank strictly smaller than the rank of $P$. Since the rank of $P$ is $O(1)$, the process must terminate after at most $O(1)$ iterations.

We are going to produce $P^{\prime}$ as follows. If $\Phi(U)$ does not have full rank, then it is contained in a hyperplane of $\mathbf{R}^{r}$. In other words, there exist integers $\alpha_{1}, \ldots, \alpha_{r}$ whose greatest common divisor is one and $\alpha_{1} m_{1}+\ldots+\alpha_{r} m_{r}=0$ for all $\left(m_{1}, \ldots, m_{r}\right)$ $\in \Phi(U)$. Let $w$ be an arbitrary element of $F$ and replace each basis vector $v_{j}$ in $P$ by $v_{j}-\alpha_{j} w$. The new progression $P^{\prime}$ will continue to contain $U$, since we have

$$
m_{1}\left(v_{1}-\alpha_{1} w\right)+\ldots+m_{r}\left(v_{r}-\alpha_{r} v_{r}\right)=m_{1} v_{1}+\ldots+m_{r} v_{r}
$$

for all $\left(m_{1}, \ldots, m_{r}\right) \in \Phi(U)$. We can assume, without loss of generality, that $\alpha_{r}$ is not divisible by $p$, the characteristic of $F$ (at least one of the $\alpha_{j}$ must be so). Select $w$ so that

$$
v_{r}-\alpha_{r} w=0 .
$$

This shows that $P^{\prime}$ has rank $r-1$. Moreover, its volume is $M_{1}, \ldots M_{r-1}$ which is at most the volume of $P$. We can use Lemma 6.3 to guarantee that $P^{\prime}$ is proper 
without increasing the rank. Furthermore, it is easy to see that (22) is still valid with respect to $P^{\prime}$. The argument is completed. Thus, from now on we can assume that $\Phi(U)$ spans $\mathbf{R}^{r}$. This information will be useful later on.

Define a highly rational number to be a number (in $\mathbf{Q}$ or $F$ ) which is of the form $a / b$ where $a, b$ are integers such that $a, b=n^{o(n)}$ and $b \neq 0$. Define a highly rational linear combination of vectors $w_{1}, \ldots, w_{k}$ to be any expression of the form $q_{1} w_{1}+\ldots+q_{k} w_{k}$ where $q_{1}, \ldots, q_{k}$ are highly rational.

We say that a set $W$ (of vectors) economically spans a set $U$ (of vectors) if there are numbers $a, b=n^{o(n)}$ such that each $u \in U$ can be represented as a highly rational linear combination of vectors in $W$ where the numerators and denominators of the coefficient are uniformly bounded from above by $a$ and $b$, respectively.

The moral of our arguments below is the following: with respect to a bounded number of operations, any linear algebraic statement which holds for "span" also holds for "economically span". This relies on the fact that any algebraic expression involving at most $O(1)$ highly rational numbers will again produce a highly rational number. In other words, the highly rational numbers behave heuristically like a subfield of $F$.

Let us now turn to the details. First we make some simple remarks about the notion of economically spanning. It is transitive in the sense that if $W$ economically spans $U$ and $U$ economically span $T$, then $W$ economically spans $T$ (but with slightly worse $o(n)$ constants). Furthermore, it is closed under union, in the sense that if $W$ economically spans $X$ and $Y$, then it economically spans the union of $X$ and $Y$. Of course we are going to use these rules only a bounded number $O(1)$ of times.

Consider the set of vectors $U:=\left\{a_{1}, \ldots, a_{n}\right\}$. We know that there is a set of vectors of cardinality $r$ which economically span $U$, namely $\left\{v_{1}, \ldots, v_{r}\right\}$. Now we are going to make use of the fact that $\Phi(U)$ spans $\mathbf{R}^{r}$. Since each vector in $\Phi(U)$ has coordinates at most $\max _{1 \leq i \leq n} M_{i} \leq 2^{n}=n^{o(n)}$ and $r=O(1)$, it follows from Cramer's rule that $\Phi(U)$ economically spans the basic vectors $\left\{e_{1}, \ldots, e_{r}\right\}$. As the map $\Phi$ is bijective ( $P$ is proper), this means $U$ economically spans $\left\{v_{1}, \ldots, v_{r}\right\}$.

We now claim that in fact there is a single element $v_{1}$ which economically spans $U$. This clearly implies rational commensurability.

Let $s$ be the smallest number such that there is a subset of size $s$ of $v_{1}, \ldots, v_{r}$ economically spanning $U$. Without loss of generality, we assume that $v_{1}, \ldots, v_{s}$ spans $U$ and write

$$
a_{i}=\sum_{j=1}^{s} c_{i j} v_{j}
$$

where $c_{i j}$ are highly rational. Let us now consider two cases:

- The matrix $C=\left(c_{i j}\right)$ has rank one. In this case the numbers $a_{i} / a_{j}$ are highly rational (as all the $c_{i j}$ are). But $\Phi(U)$ has full rank in $R^{r}$ and economically spans $v_{1}, \ldots, v_{r}$, so the $v_{i} / v_{j}$ are also highly rational. This means that $v_{1}$ (or any of the $v_{i}$, for that matter) economically spans $v_{1}, \ldots, v_{r}$, and hence also $U$ by transitivity.

- The matrix $C$ has rank larger than one. Recall that $\left(a_{1}, \ldots, a_{n}\right)$ is the normal vector of a hyperplane spanned by \pm 1 vectors. Thus, there is a \pm 1 vector $w$ which is orthogonal to $a=\left(a_{1}, \ldots, a_{n}\right)$, but not orthogonal to $C$. Express the inner product of $a$ and $w$ as a linear combination of $v_{1}, \ldots, v_{s}$ using (37). 
Observe that all of the coefficients are highly rational. Moreover, the value of the combination is 0 , but there is at least one non-zero coefficient. This implies that we can express one of $v_{1}, \ldots, v_{s}$ as a linear combination of the others with highly rational coefficients and this contradicts the minimality of $s$.

The proof of the Structure Theorem is complete.

\section{Appendix: Proof of Lemma 6.3}

In this section we give a proof of Lemma 6.3. This result appears to be "folklore", being discovered independently by Gowers and Walters (private communication) and Ruzsa (private communication), but it does not appear to be explicitly in the literature.

We first need some notation and tools from the geometry of numbers. The first tool is the following theorem of Mahler on bases of lattices, which is a variant of Minkowski's second theorem (and is in fact proven using this theorem).

Lemma 9.1. Let $\Gamma$ be a lattice of full rank in $\mathbf{R}^{d}$ (i.e., a discrete additive subgroup of $\mathbf{R}^{d}$ with d linearly independent generators). Then there exist linearly independent vectors $w_{1}, \ldots, w_{d} \in \Gamma$ which generate $\Gamma$ and such that

$$
\left|w_{1}\right| \ldots\left|w_{d}\right| \leq(C d)^{C d} \operatorname{mes}\left(\mathbf{R}^{d} / \Gamma\right)
$$

where $\operatorname{mes}\left(\mathbf{R}^{d} / \Gamma\right)$ is the volume of a fundamental domain of $\Gamma$ and $C>0$ is an absolute constant.

Proof. (Sketch) Note that the standard Minkowski basis $v_{1}, \ldots, v_{d}$ of $\Gamma$ (with respect to the unit ball $B_{d}$ ) will obey (38) but need not generate $\Gamma$ (consider for instance the lattice generated by $\mathbf{Z}^{d}$ and $\left(\frac{1}{2}, \ldots, \frac{1}{2}\right)$ for dimensions $\left.d \geq 5\right)$. However, a simple algorithm of Mahler gives a genuine basis $w_{1}, \ldots, w_{d}$ of $\Gamma$ such that $w_{j}=t_{j, 1} v_{1}+\ldots+t_{j, j} v_{j}$ for some real scalars $\left|t_{j, i}\right| \leq 1$, which thus also obeys (38) with some degradation in the $(C d)^{C d}$ factor. See, for instance [3, Chapter 8] for more details.

If $d \geq 1$ is an integer and $M=\left(M_{1}, \ldots, M_{d}\right)$ and $N=\left(N_{1}, \ldots, N_{d}\right)$ are elements of $\mathbf{R}^{d}$ such that $M_{i} \leq N_{i}$ for all $1 \leq i \leq d$, we use $[M, N]$ to denote the discrete box

$$
[M, N]:=\left\{\left(n_{1}, \ldots, n_{d}\right) \in \mathbf{Z}^{d}: M_{i} \leq n_{i} \leq N_{i} \text { for all } 1 \leq i \leq d\right\} .
$$

Also, if $G$ is an additive group, $n=\left(n_{1}, \ldots, n_{d}\right) \in \mathbf{Z}^{d}$, and $v=\left(v_{1}, \ldots, v_{d}\right) \in G^{d}$, we use $n \cdot v \in G$ to denote the quantity $n \cdot v=n_{1} v_{1}+\ldots+n_{d} v_{d}$, where of course we use $n x:=x+\ldots+x$ to denote the $n$-fold sum of $x$ (with the obvious modification if $n$ is negative). We also use $[M, N] \cdot v \subseteq G$ to denote the set $[M, N] \cdot v:=\{n \cdot v$ : $n \in[M, N]\}$. Note that a progression of rank $d$ in $G$ is nothing more than a set of the form $a+[0, N] \cdot v$ for some $a \in G$, some $d$-tuple $N=\left(N_{1}, \ldots, N_{d}\right) \in \mathbf{Z}_{+}^{d}$, some other $d$-tuple $v=\left(v_{1}, \ldots, v_{d}\right) \in G^{d}$.

We now give a "discrete John's theorem" which shows that the intersection of a convex symmetric body (i.e., a bounded open convex symmetric subset of $\mathbf{R}^{d}$ ) and a lattice of full rank is essentially equivalent to a progression.

Lemma 9.2 (Discrete John's theorem). Let $B$ be a convex symmetric body in $\mathbf{R}^{d}$, and let $\Gamma$ be a lattice in $\mathbf{R}^{d}$ of full rank. Then there exists a d-tuple

$$
w=\left(w_{1}, \ldots, w_{d}\right) \in \Gamma^{d}
$$


of linearly independent vectors in $\Gamma$ and a d-tuple $N=\left(N_{1}, \ldots, N_{d}\right)$ of positive integers such that

$$
(-N, N) \cdot w \subseteq B \cap \Gamma \subseteq\left(-d^{C d} N, d^{C d} N\right) \cdot w
$$

where $C$ is an absolute constant.

Proof. We first observe using John's theorem [10] and an invertible linear transformation that we may assume without loss of generality that $B_{d} \subseteq B \subseteq d \cdot B_{d}$, where $B_{d}$ is the unit ball in $\mathbf{R}^{d}$. We may assume $d \geq 2$ since the claim is easy otherwise.

Now let $w=\left(w_{1}, \ldots, w_{d}\right)$ be as in Lemma 9.1. For each $j$, let $N_{j}$ be the least integer greater than $1 / d\left|w_{j}\right|$. Then from the triangle inequality we see that $\left|n_{1} w_{1}+\ldots+n_{d} w_{d}\right|<1$ whenever $\left|n_{j}\right|<N_{j}$, and hence $(-N, N) \cdot w$ is contained in $B_{d}$ and hence in $B$.

Now let $x \in B \cap \Gamma$. Since $w$ generates $\Gamma$, we have $x=n_{1} w_{1}+\ldots+n_{d} w_{d}$ for some integers $n_{1}, \ldots, n_{d}$; since $B \subseteq d \cdot B_{d}$, we have $|x| \leq d$. Applying Cramer's rule to solve for $n_{1}, \ldots, n_{d}$ and (38), we have

$$
\begin{aligned}
\left|n_{j}\right| & =\frac{\left|x \wedge w_{1} \ldots w_{j-1} \wedge w_{j+1} \wedge w_{d}\right|}{\left|w_{1} \wedge \ldots \wedge w_{d}\right|} \\
& \leq \frac{|x|\left|w_{1}\right| \ldots\left|w_{d}\right|}{\left|w_{j}\right|\left|w_{1} \wedge \ldots \wedge w_{d}\right|} \\
& \leq d d^{C d} /\left|w_{j}\right| \\
& \leq d^{C^{\prime} d} N_{j},
\end{aligned}
$$

and hence $x \in\left(-d^{C d} N, d^{C d} N\right) \cdot w$ as desired.

We can now prove Lemma 6.3, which we restate here with slightly different notation.

Lemma 9.3. There is a constant $C_{0}$ such that the following holds. Let $P$ be a symmetric progression of rank $d$ in an abelian group $G$, such that every non-zero element of $G$ has order at least $d^{C_{0} d^{3}}|P|$. Then there exists a symmetric proper progression $Q$ of rank at most d containing $P$ and

$$
|Q| \leq d^{C_{0} d^{3}}|P|
$$

Proof. This claim is analogous to the basic linear algebra statement that every linear space spanned by $d$ vectors is equal to a linear space with a basis of at most $d$ vectors. Recall that the proof of that linear algebra fact proceeds by a descent argument, showing that if the $d$ spanning vectors were linearly dependent, then one could exploit that dependence to "drop rank" and span the same linear space with $d-1$ vectors.

We may assume that $P$ has the form $P=[-N / 2, N / 2] \cdot v$ for some $N=$ $\left(N_{1}, \ldots, N_{d}\right)$ and $v=\left(v_{1}, \ldots, v_{d}\right)$. We induct on $d$. The case $d=1$ is easy. Now suppose inductively that $d \geq 2$, and the claim has already been proven for $d-1$ (for arbitrary groups $G$ and arbitrary progressions $P$ ). Thanks to the induction hypothesis, we can assume that $P$ is non-proper and all $N_{i}$ are at least one. As $P$ is not proper, there is $n \neq n^{\prime} \in[-N / 2, N / 2]$ such that

$$
n \cdot v=n^{\prime} \cdot v \text {. }
$$

Let $\Gamma_{0} \subseteq \mathbf{Z}^{d}$ denote the lattice $\left\{m \in \mathbf{Z}^{d}: m \cdot v=0\right\}$. Then it follows that $\Gamma_{0} \cap[-N, N]$ contains at least one non-zero element, namely $n^{\prime}-n$. 
Let $m=\left(m_{1}, \ldots, m_{d}\right)$ be a non-zero irreducible element of $\Gamma_{0} \cap[-N, N]$ (i.e., $m / n \notin \Gamma_{0}$ for any integer $\left.n>1\right)$. By definition

$$
m \cdot v=m_{1} \cdot v_{1}+\ldots+m_{d} \cdot v_{d}=0 .
$$

We now claim that $m$ is irreducible in $\mathbf{Z}^{d}$ (i.e., that $m_{1}, \ldots, m_{d}$ have no common divisor). Indeed, if $m$ factored as $m=n \tilde{m}$ for some $n>1$ and $\tilde{m} \in \mathbf{Z}^{d}$, then $\tilde{m} \cdot v$ would be a non-zero element of $G$ of order $n \leq|P|$, contradicting the hypothesis.

Our plan is to contain $P$ inside a symmetric progression $Q$ of rank $d-1$ and cardinality

$$
|Q| \leq d^{C d^{2}}|P| .
$$

If we can achieve this, then by the induction hypothesis we can contain $Q$ inside a proper symmetric progression $R$ of rank at most $d-1$ and cardinality

$$
|R| \leq(d-1)^{C_{0}(d-1)^{3}}(C d)^{C d^{2}}|P| .
$$

If $C_{0}$ is sufficiently large (but still independent of $d$ ), then the right hand side is at most $d^{C_{0} d^{3}}|P|$, and we have closed the induction hypothesis.

It remains to cover $P$ by a symmetric progression of rank at most $d-1$ with the bound (40). Observe that $m$ lies in $[-N, N]$, so the rational numbers

$$
m_{1} / N_{1}, \ldots, m_{d} / N_{d}
$$

lie between -1 and 1 . Without loss of generality we may assume that $m_{d} / N_{d}$ has the largest magnitude, namely

$$
\left|m_{d}\right| / N_{d} \geq\left|m_{i}\right| / N_{i}
$$

for all $1 \leq i \leq d$. By replacing $v_{d}$ with $-v_{d}$ if necessary, we may also assume that $m_{d}$ is positive.

To exploit the cancellation in (39), we introduce the rational vector $q \in \frac{1}{m_{d}} \cdot \mathbf{Z}^{d-1}$ by the formula

$$
q:=\left(-\frac{m_{1}}{m_{d}}, \ldots,-\frac{m_{d-1}}{m_{d}}\right) .
$$

Since $\operatorname{gcd}\left(m_{1}, \ldots, m_{d}\right)=1$, we see for any integer $n$ that $n \cdot q$ lies in $\mathbf{Z}^{d-1}$ if and only if $n$ is a multiple of $m_{d}$.

Next, let $\Gamma \subset \mathbf{R}^{d-1}$ denote the lattice $\Gamma:=\mathbf{Z}^{d-1}+\mathbf{Z} \cdot q$. Since $q$ is rational, this is indeed a full rank lattice. We define the homomorphism $f: \Gamma \rightarrow Z$ by the formula

$$
f\left(\left(n_{1}, \ldots, n_{d-1}\right)+n_{d} q\right):=\left(n_{1}, \ldots, n_{d}\right) \cdot v .
$$

The condition (39) ensures that this homomorphism is indeed well defined, in the sense that different representations $w=\left(n_{1}, \ldots, n_{d-1}\right)+n_{d} q$ of the same vector $w \in \Gamma$ give the same value of $f(w)$. We also let $B \subseteq \mathbf{R}^{d-1}$ denote the convex symmetric body

$$
B:=\left\{\left(t_{1}, \ldots, t_{d-1}\right) \in \mathbf{R}^{d-1}:-3 N_{j}<t_{j}<3 N_{j} \text { for all } \leq j \leq d-1\right\} .
$$

We now claim the inclusions

$$
P \subseteq f(B \cap \Gamma) \subseteq 5 P-5 P .
$$

To see the first inclusion, let $n \cdot v \in P$ for some $n \in[0, N]$. Then we have $n \cdot v=$ $f\left(\left(n_{1}, \ldots, n_{d-1}\right)+n_{d} q\right)$. From (41) we see that the $j^{\text {th }}$ coefficient of $\left(n_{1}, \ldots, n_{d-1}\right)+$ $n_{d} q$ has magnitude at most $3 N_{j}$, and thus $n \cdot v$ lies in $f(B \cap \Gamma)$ as claimed. To see the second inclusion, let $\left(n_{1}, \ldots, n_{d-1}\right)+n_{d} q$ be an element of $B \cap \Gamma$. By subtracting 
an integer multiple of $m_{d}$ from $n_{d}$ if necessary (and thus adding integer multiples of $m_{1}, \ldots, m_{d-1}$ to $\left.n_{1}, \ldots, n_{d-1}\right)$, we may assume that $\left|n_{d}\right| \leq\left|m_{d}\right| / 2$. By (41) and the definition of $B$, this forces $\left|n_{j}\right| \leq 5 N_{j}$ for all $1 \leq j \leq d$, and hence

$$
f\left(\left(n_{1}, \ldots, n_{d-1}\right)+n_{d} q\right)=\left(n_{1}, \ldots, n_{d}\right) \cdot v \subseteq[-5 N, 5 N] \cdot v=5 P-5 P .
$$

Next, we apply Lemma 9.2 to find vectors $w_{1}, \ldots, w_{d-1} \in \Gamma$ and $M_{1}, \ldots, M_{d-1}$ such that

$$
(-M, M) \cdot w \subseteq B \cap \Gamma \subseteq\left(-d^{C d} M, d^{C d} M\right) \cdot w .
$$

Applying the homomorphism $f$, we obtain

$$
(-M, M) \cdot f(w) \subseteq f(B \cap \Gamma) \subseteq\left(-d^{C d} M, d^{C d} M\right) \cdot f(w)
$$

where $f(w):=f\left(w_{1}\right), \ldots, f\left(w_{d-1}\right)$. Observe that $\left(-d^{C d} M, d^{C d} M\right) \cdot f(w)$ is a symmetric progression of rank $d-1$ which contains $f(B \cap \Gamma)$ and hence contains $P$. Also, since $\left(-d^{C d} M, d^{C d} M\right) \cdot f(w)$ can be covered by $O\left((C d)^{C d^{2}}\right)$ translates of $(-M, M) \cdot f(w)$, and similarly $5 P-5 P$ can be covered by $O\left(C^{d}\right)$ translates of $P$, we have

$$
\begin{aligned}
\left|\left(-d^{C d} M, d^{C d} M\right) \cdot f(w)\right| & \leq(C d)^{C d^{2}}|f(B \cap \Gamma)| \\
& \leq(C d)^{C d^{2}}|5 P-5 P| \\
& \leq(C d)^{\left.C d^{2}\right)} C^{d}|P|
\end{aligned}
$$

which proves (40). This completes the induction and proves the lemma.

Remark 9.4. It is not hard to remove the hypothesis that $G$ is "nearly torsion free" in the sense that the order of every non-zero element is much larger than $P$; however one must then drop the conclusion that $Q$ is symmetric (this can be seen simply by considering the case $G=\mathbf{Z} / 2 \mathbf{Z}$ ).

\section{REFERENCES}

[1] Y. Bilu, Structure of sets with small sumset. Structure theory of set addition. Astérisque, No. 258 (1999), xi, 77-108. MR1701189 (2000h:11109)

[2] Y. Bilu, V. Lev, I. Ruzsa, Rectification principles in additive number theory, Discrete Comput. Geom. 19 (1998), 343-353. MR1608875 (2000a:11018)

[3] J. W. S. Cassels, An introduction to the geometry of numbers, Springer, Berlin, 1959. MR0157947 (28:1175)

[4] M. Chang, A polynomial bound in Freiman's theorem, Duke Math. J. 113 (2002), no. 3, 399-419. MR.1909605 (2003d:11151)

[5] P. Erdös, On a lemma of Littlewood and Offord, Bull. Amer. Math. Soc. 51 (1945), 898-902. MR.0014608 (7:309j)

[6] G. Freiman, Foundations of a structural theory of set addition. Translated from the Russian. Translations of Mathematical Monographs, Vol 37. American Mathematical Society, Providence, R. I., 1973. vii+108 pp. MR0360496 (50:12944)

[7] B. Green, I. Ruzsa, Sets with small sumset and rectification, Bull. London Math. Soc. 38 (2006), 43-52. MR2201602 (2006i:11027)

[8] B. Green, I. Ruzsa, Freiman's theorem in an arbitrary abelian group, preprint.

[9] G. Halász, Estimates for the concentration function of combinatorial number theory and probability, Period. Math. Hungar. 8 (1977), no. 3-4, 197-211. MR0494478 (58:13338)

[10] F. John, Extremum problems with inequalities as subsidiary conditions, Studies and essays presented to $R$. Courant on his 60th birthday, Jan 8. 1948, Interscience Publishers Inc., New York, NY, 1948, 187-204. MR0030135(10:719b)

[11] J. Kahn, J. Komlós, E. Szemerédi, On the probability that a random \pm 1 matrix is singular, J. Amer. Math. Soc. 8 (1995), 223-240. MR.1260107 (95c:15047) 
[12] J. Komlós, On the determinant of (0,1) matrices, Studia Sci. Math. Hungar. 2 (1967), 7-22. MR0221962 (36:5014)

[13] J. Komlós, On the determinant of random matrices, Studia Sci. Math. Hungar. 3 (1968), 387-399. MR0238371 (38:6647)

[14] A. Odlyzko, On subspaces spanned by random selections of \pm 1 vectors, J. Combin. Theory Ser. A 47 (1988), no. 1, 124-133. MR924455 (89h:05012)

[15] I. Ruzsa, Generalized arithmetical progressions and sumsets, Acta Math. Hungar. 65 (1994), no. 4, 379-388. MR 1281447 (95k:11011)

[16] I. Ruzsa, An analog of Freiman's theorem in groups, Structure theory of set addition, Astérisque, No. 258 (1999), 323-326. MR.1701207 (2000h:11111)

[17] T. Tao and V. Vu, On random \pm 1 matrices: Singularity and Determinant, Random Structures and Algorithms 28 (2006), no 1, 1-23. MR2187480 (2006g:15048)

Department of Mathematics, UCLA, Los Angeles, California 90095-1555

E-mail address: tao@math.ucla.edu

Department of Mathematics, Rutgers University, Piscataway, New Jersey 08854-8019

E-mail address: vanvu@ucsd.edu 